\title{
A FURTHER REPORT ON LUNG RESECTION FOR PULMONARY TUBERCULOSIS
}

\author{
BY \\ B. J. BICKFORD, F. RONALD EDWARDS, J. R. ESPLEN, J. G. GIFFORD, \\ AND O. F. THOMAS
}

From the Thoracic Surgical Service of the Liverpool Region (Aintree, Broadgreen, and Cleaver Hospitals) and the Department of Surgery, University of Liverpool

(RECEIVED FOR PUBLICATION SFPTEMBER 30, 1952)

Eighteen months ago we published an account of lung resection for pulmonary tuberculosis (Thorax, 1951, 6, 25). The following communication is a reassertion of the general principles of this form of treatment, a record of our further experiences in 729 cases, and a follow-up of all our cases of pulmonary tuberculosis that have had a resection two years or more previously.

Some confusion still exists not only concerning the type of tuberculous disease that should be resected but of the time when the resection operation should be done, and we would make it clear that certain guiding principles govern our selection of cases for this type of operation.

Resection of lung tissue affected by tuberculosis is carried out for two reasons: (1) To remove an area of disease which remains persistently active despite bed rest, chemotherapy, and simple relaxation therapy; (2) to remove an area of disease which has reached a stage of apparent "quiescence," but is nevertheless of a type that experience shows to be in not inconsiderable danger of reactivation under conditions of stress and intercurrent infection. Resection is an insurance for the future.

Resection is only carried out when the body defences against the disease have been mobilized as fully as possible. The maximum localization of the disease is obtained by simple means before resection is undertaken. Most cases of pulmonary tuberculosis after treatment as above have one or more areas of confluent disease together with scattered small foci. The confluent areas are assessed as to the likelihood of final healing. If this is doubtful they are considered for resection. The small scattered foci are unlikely to reactivate, provided that hyperexpansion of the remaining lung tissue is not permitted.

It is recognized that at the stage of onset tuberculosis may be a generalized disease, and that resection of any primary pulmonary infection io not indicated unless, after a long period of time@ activity of the lesion persists despite all othe forms of treatment.

The continued activity of a caseous pneumonia following rupture of an intrabronchial gland ma N require removal of the affected area of the lung.

The chances of children controlling their disease by simple methods is very high, and resection of lung tissue under the age of 16 is only carried ou where the disease has remained active over a lon period. Between the ages of 16 and 20 there is considerable instability of the disease, and resectio $\overrightarrow{\vec{\Phi}}$ is only carried out between these ages after carefu assessment.

After resection the remaining lung tissue of either side of the chest must not be permitted to expand beyond its normal size. This entails re ducing the volume of the hemithorax by elevation of the diaphragm and/or resection of ribs.

Tuberculous endobronchitis at the proposed lin of section of the bronchus must be cleared a? much as possible by chemotherapy before resection. Resection is contraindicated while tuber culous ulceration, granulation tissue, or oedem 8 persists at the line of section, but simple rednes? of the mucosa does not obviate operation.

The use of chemotherapy to control the acute phase or an exacerbation of the disease takes pre cedence over its use as a cover for operation although it is routinely used during the operation

INDIVIDUAL Forms OF THE Disease

CASEOUS Nodules.- If these lesions are more than $1 \mathrm{~cm}$. in diameter and persist longer than year we advise excision. Many are closed; other帝 have small and usually intermittent bronchial conf munications. If smears are made of the contente of the nodule, they will almost always show high concentration of tubercle bacilli. We cong 
sider that this form of the disease is the reaction of an individual with a high resistance to a tuberculous infection. A number of these nodules will ultimately empty out into the bronchus with subsequent healing of the focus, others will calcify, but many will be a cause of continued concern with periods of exacerbation and regression. Unfortunately there is no method of assessing which will heal in the course of time and which will not. We have seen a number of well-calcified nodules break down and produce extensions of the disease. Caseous nodules can be simply removed by segmental resection, preventing rupture of this aggregation of tubercle bacilli into the bronchial tree, with its dangers not only to the patient but to others.

Fibro-caseous Disease With or Without CAVitation.-We have seen no cause to alter our original views on the treatment of this form of disease. We would again emphasize the importance of preventing hyperexpansion of the remaining lung tissue after resection of tuberculous areas. In our earlier group of cases upper lobectomy was done without a corrective thoracoplasty. We have had 10 cases of reactivation of small caseous areas left behind in the apical lower segment, which were judged to be healed or nearly healed at the time of operation. These areas had suffered a severe degree of hyperexpansion, and the subsequent reactivation has suggested that the effect on the tuberculous area has been to light the infection. The reactivation occurred within 18 months of the operation. We have not seen a reactivation under similar circumstances where a corrective thoracoplasty has been done.

After a cautious start we have made increasing use of segmental resection, and with continued experience we find that this conservative operation is satisfactory where the disease is confined to segmental areas.

Except for the occasional isolated nodule at the extreme periphery of the lung we do not advocate any form of wedge resection. In many cases, although the segmental disease may be small in amount, there may be tuberculous disease in the bronchus for some distance towards the hilum. Complete removal of the segmental bronchus is thus important, for further spread of disease to a neighbouring segment from the retained diseased bronchus may follow at a later date.

Biiateral resection has been performed in 10 cases and we are considering this more frequently. To mitigate hyperexpansion of the remaining lung tissue under these circumstances we usually perform a phrenicectomy on one side and a small corrective thoracoplasty on the other. Seven cases have been treated by bilateral segmental resection and three by lobectomy on one side and segmental resection on the other.

In cases of bilateral disease, provided it is stable, patients will tolerate a resection operation on one side without any great likelihood of the opposite side deteriorating. Each case, however, will have to be judged on its merits with a full study of the natural history of the disease on each side. It is important, however, to see finality in any bilateral surgical programme and to be sure that what one embarks upon is technically and functionally possible and will leave the patient after his second operation with a reasonable chance of a cure.

UnSatisfactory ARTificial PNeUmothorax.Pathological studies of the tuberculous areas removed from patients who have had an artificial pneumothorax for some years support the view that this form of treatment is doubtfully curative in patients who have large areas of fibro-caseous disease with or without cavitation. The problem of when to abandon the pneumothorax is a difficult one, and we are coming to take the view in such cases that the pneumothorax will improve the patient's condition up to a certain point, but that in most cases final healing will only take place after the gross area of disease has been resected. The remainder of the lung is decorticated and a corrective thoracoplasty performed. A good pneumothorax that is tending to obliterate from the bottom with the diseased area well relaxed but showing signs of intermittent activity is a definite indication for a resection.

EMPYEMA.-Tuberculous infection of the pleural cavity is treated primarily by intrapleural streptomycin and P.A.S. injections combined with aspiration. If the cavity is not obliterated by this means the sac is excised. If tuberculous disease persists in the underlying lung the affected area is excised with the empyema cavity, following the same principles as for resection uncomplicated by an empyemá. Excision of the sac may be difficult, and it is frequently entered in the region of the costophrenic sulcus, so that pre-operative sterilization of the cavity should be as complete as possible. Fortunately this mishap is only rarely followed by spread of infection. Seventeen cases of tuberculous empyema with lung resection are included in the series.

Persistent Effusions.-If pleural effusions persist over a period of one year excision of the pleura should be considered together with underlying diseased lung tissue if required. These effusions are potentially unsafe and frequently 
become purulent, perforating into the lung or through the chest wall, often many years later.

FAILED ThORACOPLASTY.-Examination of lung tissue removed from under a thoracoplasty demonstrates that the final healing of the tuberculous process is frequently less secure than one would like to believe. Bronchiectatic changes are usual. In those patients where resection is required the lung is best approached by the removal of the anterior end of the fifth rib, the extrapleural plane being entered and the posterior plaques of reformed bone divided backwards and upwards so that the upper part of the chest wall can be lifted and replaced as a flap. In cases of persistent upper lobe cavities it is frequently found that the cavity is adherent to the costovertebral gutter. It can easily be torn across during removal, leaving an exposed area of tuberculous granulation tissue on the inner side of the chest wall. This can be treated by laying a pad of formalin over the granulation tissue area for some time during the course of the operation or by diathermy.

\section{TOTAL EXPERIENCE OF LUNG RESECTION FOR TUBERCULOSIS}

The experience that we have had with this form of treatment comprises 729 operations up to March, 1952.

The procedures performed have been :

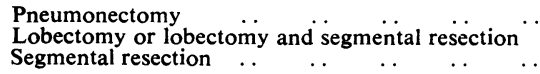

Lobectomy or lobectomy and segmental resection

The number of pneumonectomies performed has steadily fallen. In some of our earlier cases there is no doubt that we removed areas of relatively healthy lung tissue that we would now leave behind. This is because we were afraid of transgressing any lung tissue or adherent fissures for fear of producing an empyema or acute spread of disease. This fear we now know to have been greatly exaggerated, and with careful technique and chemotherapy such transgressions can be safely made.

The operation has now become a very selective and conservative procedure, and we are undertaking more and more segmental resections. We would emphasize again that caseous nodules if soft and measuring over $5 \mathrm{~mm}$. should not be left behind. In most cases the corrective thoracoplasty is performed at the same time as the resection. Over the age of 40 , where the general condition is not very good, or if there is a tendency to bronchitis or bronchospasm, it may be delayed for some weeks.
Where a segmental resection is performed it is now our practice to perform a phrenicectomy at $\frac{\bar{\sigma}}{\bar{\omega}}$ the same time as the operation. The original con- $\vec{\nabla}$ cept we held, that a pneumoperitoneum combined with a phrenic crush would allow the diaphragm $\%$ to ascend to a high level and then be fixed by $\vec{O}$ adhesions in the costophrenic sulcus, has been shown by experience to be wrong. After the $\vec{\omega}$ recovery of diaphragmatic action, the descent in most cases is complete.

There is no doubt that the various resection $v$ operations required for pulmonary tuberculosis are $\dot{\omega}$ technically more difficult to perform on the whole $\overrightarrow{0}$ than operations on the chest wall, and it has been 의 suggested that it is not possible to delegate somuch work to junior staff as has been usual with thoracoplasties. Junior staff require the assistance of an experienced surgeon for a much longer period if they are training in this type of operation, and senior staff must be prepared to give this time if the whole team is to achieve satisfactory results. Thirty-six per cent of the operations in this series? have been done by registrars.

\section{Post-operative Deaths}

Deaths within three months of the operation have totalled 14 , giving a post-operative mortality rate of $1.92 \%$.

Analysis of these shows:

\begin{tabular}{|c|c|c|c|}
\hline & Total & $\begin{array}{l}\text { Post-operative } \\
\text { Deaths }\end{array}$ & Percentage \\
\hline $\begin{array}{l}\text { Pneumonectomy . } \\
\text { Lobectomy } \\
\text { Segmental resection }\end{array}$ & $\begin{array}{l}178 \\
292 \\
259\end{array}$ & $\begin{array}{l}9 \\
4 \\
1\end{array}$ & $\begin{array}{l}5 \cdot 1 \\
1 \cdot 4 \\
0 \cdot 4\end{array}$ \\
\hline
\end{tabular}

PNeumonectomy.-Cases that require pneumonectomy are naturally the severer forms of the disease. In some cases the general condition is 응 poor, the operation being performed as a desperate measure to save life. Many patients also have had at some time extensive disease on the opposite side, and its complete quiescence may still be in doubt $\odot$ at the time of operation. Nearly all the oper- $N$ ations in this group have been extrapleural pneu- N monectomies, and the loss of blood under such circumstances may be considerable. Some of the operations, particularly where there has been an overlying empyema, have been tedious and prolonged. The mortality in this group is thus high.

The causes of death following pneumonectomy have been:

Acute pulmonary oedema

Fistula and empyema with progressive disease

Acute cardiac failure

Pulmonary embolism

Acute bronchopneumonia

Collapse on table 
The two cases of acute pulmonary oedema in the remaining lung still remain somewhat of a mystery as to their causation. They occurred in relatively young patients, in whom the respiratory function of the opposite side had not been in doubt and whose cardiovascular system was normal. The question of over-transfusion was checked, and, as the blood loss is calculated during the operation by weighing the swabs, a knowledge is maintained of the fluid requirements of these patients. If anything, they had received less fluid than required. The pulmonary oedema started within a few hours of the operation, and death occurred within 48 hours. Necropsy was carried out in both cases, and nothing but a severe degree of pulmonary congestion and oedema found. There was no suggestion that a spill-over of secretion had occurred.

The three patients who developed empyemata and fistulae and died with progressive disease on the opposite side were all desperate cases, one a diabetic.

The patient who died of cardiac failure had persistent attacks of paroxysmal tachycardia which became uncontrollable and death occurred on the seventh day. She had given a history of these before operation, but she had been judged fit to stand the operation. Necropsy showed no definite cardiac lesion.

One patient developed a fatal pulmonary embolism 21 days after operation and another developed an acute and apparently non-specific bronchopneumonia in the remaining lung five days after operation. Death occurred three days later.

LOвестомY.-Four patients have died after lobectomy, the reasons being as follows:

$\begin{array}{llllll}\text { Acute pulmonary oedema and cardiac failure } & . & \ldots & 1 \\ \text { Acute staphylococcal infection } & \ldots & \ldots & \ldots & \ldots & 1 \\ \text { Pulmonary embolism } & \ldots & \ldots & \ldots & \ldots & 1 \\ \text { Haemorrhage on the table } & \ldots & \ldots & \ldots & \ldots & 1\end{array}$

The patient who developed pulmonary oedema had a lobectomy from under a thoracoplasty. His pre-operative condition was poor, and he died nine days after operation with severe pulmonary oedema and cardiac failure.

Segmental Resection.-One patient died from a sudden tension pneumothorax which was unfortunately not recognized in the middle of the night.

Late Deaths.-Of the remaining 715 patients, five died after the post-operative period.

The cause of death has been:

1 (At necropsy the condition of the chest was found to be satisfactory)
With reference to the late deaths, tuberculous infection of the residual fluid in the pleural space following pneumonectomy or lobectomy can occur early or late. It is probable that in those cases where pus formation is late a mild infection has persisted from early days either from soiling at the time of operation or from damaged lymph nodes. Seven cases developed clinical signs of an empyema three or more months after operation, six after pneumonectomy and one after an upper lobectomy and apical lower segmental resection. Attention is drawn to this complication by the sudden coughing up of pus, preceded usually by a few days' malaise. In five cases out of the seven an acute tuberculous bronchopneumonia developed in parts of the remaining lung tissue. That this is a serious complication is shown by the fact that four of the seven patients have died and a further patient is seriously ill. The remaining two are reasonably well.

\section{Post-OPERATIVE Complications}

EMPYEMA.-This is the most serious complication of resection, and in all (early and late) 25 cases have occurred, 16 being associated with a bronchial fistula. Details are as shown :

$\begin{array}{llll}\text { Pneumonectomy } & \ldots & \ldots & 15\left\{\begin{array}{l}5 \text { cured } \\ 4 \text { sinuses persisting } \\ 6 \text { dead }\end{array}\right. \\ \text { Lobectomy .. } & \ldots & \ldots & 8\left\{\begin{array}{l}3 \text { cured } \\ 4 \text { sinuses persisting } \\ 1 \text { dead }\end{array}\right. \\ \text { Segmental } \ldots & \ldots & \ldots & 2\left\{\begin{array}{l}1 \text { cured } \\ 1 \text { sinus persisting }\end{array}\right.\end{array}$

Treatment has been by drainage and thoracoplasty, or a revision operation if a corrective thoracoplasty has been combined with the resection. The problem of the treatment of a combined subscapular space infection with an empyema and fistula is very great, and may be almost insoluble. (Two such cases have shown remarkable closing of these spaces following administration of isonicotinic acid hydrazide after some 18 months of stagnation.) It is fair to state that the incidence of this complication was higher in our earlier group of cases and has fallen with increasing experience.

"AIR LeaKs."-A number of patients undergoing segmental resections have continued to leak air for a considerable time after the operation. In a group of 100 consecutive segmental resections this occurred five times. This complication appears to be due to the persistent patency of small intersegmental bronchioles. On examining the stripped surface of the remaining lung tissue fine bronchi will be found upon which occasional elliptical holes will be noticed. These are the torn ends of intersegmental bronchioles. Usually they will be 
occluded by fibrin and by the expansion of the surrounding alveoli, but occasionally they continue to leak air, and if they do so the collapse of the lung will prevent their being sealed over. It is thus important that a search be made for these openings at the time of operation and that they be ligated. The consistency of the bronchial wall is like a shrimp-shell at this level, and a thread ligature can easily be applied after picking up the edges with a pair of fine artery forceps. It will be found that the persistent air leak tends to occur in those cases where the lung tissue on the two sides of the intersegmental plane has been normal. If there has been extensive disease in a segment or an inflammatory process with collapse on any occasion it is unlikely that persistent air leaks will occur, as these minute intersegmental bronchioles tend to become obliterated. This might explain the rarity of this complication after segmental resection for bronchiectasis. The management of a patient who continues to blow air after a segmental resection or any form of operation where a lung strip has been performed is as follows.

If air is being blown out on coughing at the end of 48 hours the drainage tube is left in for a further 24 hours. If air leakage still continues the drainage tube is removed and a needle (as used for a tension pneumothorax with a clamp to fix it to the chest wall) is inserted through the first intercostal space anteriorly. This is connected to an underwater seal bottle and $1 \mathrm{~g}$. of streptomycin is injected through it into the pleural cavity. The needle is removed and reinserted in a different position every 48 hours, and $1 \mathrm{~g}$. of streptomycin is injected every 48 hours into the pleural cavity. Needle drainage is maintained until the leak has closed, which will occur as the lung expands. It is useless to abandon needle drainage in the hope that closure will take place spontaneously, for the upper part of the lung will again become compressed and the tiny bronchial opening remain uncovered. The intrapleural streptomycin, besides combating infection, induces a mild pleuritis which we believe may assist in sealing the leak. The leak will usually close in seven to 10 days. If it persists over three weeks, then reopening of the chest, suture of the leak, and decortication of the lung are indicated. We have had to undertake this secondary operation in six patients out of the 259 segmental resections, and it was successful in all. The observations made above as to the pathology of these leaks have been gleaned from these six cases. If an air leak has persisted for some time and has then closed spontaneously the lung tends to be unequally expanded, the lower part being rather crowded and fixed in the lower듬 chest. The upper part of the remaining lung tissue $\frac{\bar{\sigma}}{\bar{c}}$ then has to hyperexpand to fill the chest. This is undesirable, and such cases should have either $a$ 을 small secondary corrective thoracoplasty or theen chest should be reopened and the lung decor- $\overrightarrow{0}$ ticated. After segmental resection there have beentwo cases of empyema, both treated by drainage $\vec{\sigma}$ and corrective thoracoplasty.

Clotted HaEmothoraX.-After lobectomy and ${ }^{x}$ segmental resection this complication may seriously affect function. The bleeding appears to be usually due to reactionary haemorrhage from divided adhesions on the chest wall.

Aspiration is difficult and frequently impossible. $\vec{\nabla}$ Pyrexia may persist during the stage of absorption of haemoglobin. Expansion of the lung to cover尺̊ the divided end of the bronchus is interfered with? and the possibility of fistula developing is increased. Severe pleural thickening and interference with $\vec{\bullet}$ lung expansion will persist.

In two cases the chest has been reopened and the clot removed. This has been followed by fullo and satisfactory lung expansion. More recently streptokinase-streptodornase has been found to be of value, but we have not given it before the tenth $\frac{}{\mathrm{O}}$ post-operative day. If there is difficulty in treating a clotted haemothorax by simple means, then thereö should be no hesitation in reopening the chest, for there is nothing to lose and much to gain.

\section{SPREAD OF Disease}

There have been 10 cases (already described) where there has been an aspiration tuberculous bronchopneumonia due to the development of an: empyema with a secondary bronchial fistula. Excluding these, in only two cases in the wholeo series have we found new areas of disease on the $₹$ opposite side or the same side which might be의 deemed a spill-over of tuberculous material. One $>$ of us (F.R.E.) operates always with the patient in the lateral head-down position, which, combinedñ with tracheal suction as required, appears to give reasonable protection to the opposite lung, but another (B.J.B.) frequently uses the face-down position for "wet" pneumonectomies. The removal of the severely diseased areas of lungo tissue prevents the aspiration of heavily infected $\bar{C}$ secretion into other parts of the lung during the? post-operative stage.

\section{Reactivation of Old Disease}

A main cause of reactivation of disease in the $\frac{\overparen{P}}{\mathbb{D}}$ series appears to be severe hyperexpansion of lung $\varrho$ tissue. We have already mentioned an increase in size of retained caseous nodules or areas of localo 
spread occurring in 10 cases. Six have been treated by further surgery, three having a resection of the apical segment of the lower lobe with a corrective thoracoplasty, and three a thoracoplasty only. Ten other patients developed a reactivation, usually quite small, of disease on the opposite side. Most of the patients with reactivation seem to be those who refuse to follow any organized treatment.

\section{Note on ANAESTHESIA}

No major changes in anaesthetic technique have been introduced since the beginning of the series.

Following induction with thiopentone, anaesthesia is maintained with nitrous oxide-oxygen supported by small supplementary doses of barbiturate. Natural respiration is suspended by the use of gallamine triethiodide and $d$-tubocurarine chloride, and pulmonary ventilation is maintained automatically by machine. For control of secretions we continue to rely on pre-operative physiotherapy and on the employment of a head-down tilt and suction at operation, the suction catheter being used with care and discretion so that the bronchial epithelium distal to the carina is interfered with as little as possible. Before leaving the theatre the patients are expected to be awake but analgesic and capable of coughing effectively when asked to do so.

\section{TWO-YEAR Follow-UP}

A follow-up was made in July, 1952, of all cases that had been treated by resection two years or more previously. They number 223 and the length of time since operation is as follows :

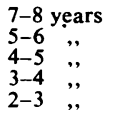

$\begin{array}{rrrrrr}\ldots & \ldots & \ldots & \ldots & \ldots & 1 \\ . . & \ldots & \ldots & \ldots & \ldots & 3 \\ \ldots & \ldots & \ldots & \ldots & \ldots & 5 \\ . . & \ldots & \ldots & \ldots & \ldots & 47 \\ & & & & & 167\end{array}$

Analysis of the present position shows :

\begin{tabular}{|c|c|c|c|c|c|c|c|}
\hline & No. & 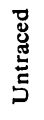 & 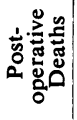 & $\begin{array}{c}\text { Late } \\
\text { Deaths }\end{array}$ & 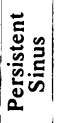 & $\begin{array}{c}\text { Evidence } \\
\text { of } \\
\text { Active } \\
\text { Disease }\end{array}$ & $\overline{0}$ \\
\hline Pneumonectomy.. & 100 & 1 & 4 & $\begin{array}{c}4 \\
\text { (one } \\
\text { non-TB) }\end{array}$ & 4 & 2 & $\overline{85}$ \\
\hline $\begin{array}{l}\text { Lobectomy or lob- } \\
\text { ectomy and seg- } \\
\text { mental resection }\end{array}$ & 105 & 0 & 1 & $\mathbf{0}$ & 0 & 8 & 96 \\
\hline Segmental resection & 18 & 0 & 0 & 0 & 1 & 1 & 16 \\
\hline Total & $\overline{223}$ & 1 & 5 & 4 & 5 & 11 & $\overline{197}$ \\
\hline
\end{tabular}

Late Deaths.-One patient died from a septic abortion, and necropsy showed no evidence of active tuberculous disease. The other three patients died following a late rupture of a tuber-. culous empyema into the bronchial tree with an aspiration dissemination of tuberculosis throughout the opposite lung occurring seven, 12 , and 26 months respectively after pneumonectomy.

Persistent SinUs.-Five patients have an unhealed sinus in the chest wall.

(1) Pleuro-pneumonectomy for tuberculous empyema with bronchial fistula. Active disease present in opposite lung. Condition fair.

(2) Pneumonectomy after failed thoracoplasty. Condition fair. Other lung healthy.

(3) Pneumonectomy for destroyed lung. Other lung healthy. Condition good.

(4) Pleuro-pneumonectomy for tuberculous empyema and destroyed lung. Other lung healthy. Condition fair.

(5) Apico-posterior segmental resection left upper lobe. Remaining lung tissue appears healthy. Condition fair. Undergoing further plastic treatment at the moment.

Evidence of Active Disease.-Eleven patients are presenting either a positive sputum or radiological evidence to suggest that the tuberculous infection is not on the way to healing.

(1) Left pneumonectomy for extensive cavitating disease. Early bronchopleural fistula and empyema treated by thoracoplasty and closed. Small infiltration right lung: ? healing now. Right lung clear post-operatively.

(2) Left pneumonectomy for destroyed lung. In mental hospital. Developed small nodule under right clavicle. Not progressing. Pre-operatively right lung clear.

(3) Left upper lobectomy. Peripheral cavities with haemoptysis. Has had intermittent positive sputum since operation, the cause for which has not been found despite the fullest examination. General condition very good. Working.

(4) Left upper lobectomy for cavitating disease. A few nodules left in apical lower segment judged to be healing. No corrective thoracoplasty. Reactivation in these nodules. To have further surgical treatment.

(5) Right upper lobectomy for failed thoracoplasty. Slow but progressive disease through remainder of right lung and in left lung. Appears to have little resistance.

(6) Right upper lobectomy for cavitating disease. Well for two years. Now readmitted to sanatorium with some reactivation of disease on left side in which fibrotic areas had been noted before operation.

(7) Right upper lobectomy for caseous disease. Nodules noted in apical lower segment but judged as healing. No thoracoplasty. Twenty-one months later reactivation occurred from these nodules. Having medical treatment and awaiting surgery.

(8) Right upper lobectomy and apical lower segmental resection for cavitating disease. Took own 
discharge four weeks after operation. Nine months later extension of disease throughout remainder of right lung. Patient refused further treatment.

(9) Left upper lobectomy for cavitating disease. Aged 17. Progressive disease in left lower lobe 18 months later. Undergoing medical treatment before further surgery. This patient was operated upon too early in the course of his disease.

(10) Apico-posterior segmental resection of the left upper lobe for cavitating disease. Few small nodules in apical segment of left lower lobe noted at operation, but judged to be healing and left. Sputum intermittently positive since operation. Probably reactivation in apical lower nodules.

(11) Right upper lobectomy. Developed acute bilateral tuberculosis one year after operation. Condition fair only.

The untraced patient was known to be well six months after operation.

Of the 223 patients, 21 patients $(9 \%)$ have either died of pulmonary tuberculosis or show some evidence of activity at follow-up. One hundred and ninety-seven patients $(88 \%)$ are well and show no clinical, radiological, or bacteriological evidence of active disease two years or more after operation.

\section{Present Position of Pulmonary Resection for TUBERCULOSIS}

It is interesting to consider the type of case on which we have performed therapeutic thoracoplasty during the last three years. Few of these operations have been done and the circumstances have been as follows.

Two early extrapleural pneumonectomies were abandoned because of difficulties in sc, yarating the pleura and a thoracoplasty substituted. It is likely that with our further experience of this operation we would have now found the operation technically feasible.

On occasion, on opening the chest to undertake a lobectomy, a mass of scattered nodules, some soft and approaching $1 \mathrm{~cm}$. in diameter, were found throughout the remainder of the lung. This had been unsuspected on radiography. One had felt that a pneumonectomy would be required to eradicate such extensive disease, but a similar process might be present in the opposite side without any clear radiological evidence. Under such circumstances we have continued the operation as the first stage of a thoracoplasty.

There have been a few therapeutic thoracoplasties on cavitating disease in patients with extensive infiltrations on the opposite side. This is the only group in which the thoracoplasty has been done by design.
No attempt is being made at this stage to come pare lung resection with the long-established form of collapse therapy as a procedure for curing pue monary tuberculosis. "Cure" is a word that has been used with great hesitancy by those engagee in the treatment of pulmonary tuberculosis, an $\$$ this hesitancy has been an indication of doubt ity the final efficacy of the methods of treatmerif hitherto adopted. These methods have relie年 entirely on the natural cure by the body defences, supplemented frequently by collapse procedures which enable them to act more efficiently. Resecto tion is a direct attack on the more massive areas of the disease which are the most difficult for the body to sterilize. The period of study of our cases. is as yet short, and a firmer opinion of the valug of resection will only be obtained when a large number of these cases have been followed for five years at least. In pulmonary tuberculosis, as i any other disease, the complete cure of a case must be the aim of treatment. The removal of doubtfư areas in the lung where we know the tubercle bacillus can remain dormant for many yearg appears to be a further step towards the achieves ment of this cure, provided that no serious sequelae of the removal of lung tissue supervens in the long run. Will the functional effect of los of lung tissue be more serious than collapse of the lung in the long-distant future? Does the remova of the main areas of the disease reduce the resis tance of potentially susceptible people to furthep. infection? These are questions that can only b) answered after a long period.

\section{Summary}

A reassertion is made of the principles under lying the treatment of pulmonary tuberculosis b更 resection.

Seven hundred and twenty-nine cases had been treated by March, 1952. Fourteen patient $(1.92 \%)$ died within three months of operation.

Pleural infection occurred in 25 cases.

Air leaks after segmental resection are prob N ably due to persistent patency of intersegmenta出 bronchioles. Treatment of this complication is indicated.

A follow-up of 223 patients operated on twक्षे years or more previously shows that 197 patient\$? $(88 \%)$ are well and free of activity.

Our most grateful thanks are due to the many ches, physicians of the Liverpool Region and elsewher $\overrightarrow{0}$ who have helped us with the follow-up, and to Mrs. Anthony, our secretary at Aintree Hospital. 